\title{
Transcriptome analysis of pancreatic cancer cell response to treatment with grape seed proanthocyanidins
}

\author{
WEIHUA WANG ${ }^{1,2}$, LEILEI ZHAN ${ }^{3}$, DONGQI GUO ${ }^{1,2}$, YANJU XIANG ${ }^{1,2}$, \\ YU ZHANG $^{3}$, MUXING TIAN $^{1,2}$ and ZHANJIANG HAN ${ }^{1,4}$ \\ ${ }^{1}$ Department of Food Science, College of Life Sciences, Tarim University; \\ ${ }^{2}$ Xinjiang Production and Construction Corps Key Laboratory of Deep Processing of Agricultural Products \\ in South Xinjiang, Alar, Xinjiang 843300; ${ }^{3}$ Center for Genome Analysis, ABLife Inc., Wuhan, Hubei 430075; \\ ${ }^{4}$ Xinjiang Production and Construction Corps Key Laboratory of Protection and Utilization of \\ Biological Resources in Tarim Basin, Alar, Xinjiang 843300, P.R. China
}

Received February 22, 2018; Accepted October 25, 2018

DOI: $10.3892 / \mathrm{ol} .2018 .9807$

\begin{abstract}
Grape seed proanthocyanidins (GSPs) have been demonstrated to exhibit potential chemotherapeutic efficacy against various cancer types. To determine the underlying molecular mechanisms involved in GSP-induced apoptosis, the present study prepared pancreatic cancer (PC) cells samples, $\mathrm{S} 3, \mathrm{~S} 12$ and S24, which were treated with $20 \mu \mathrm{g} / \mathrm{ml}$ GSPs for 3, 12 and 24 h, respectively. Control cell samples, C3, C12 and C24, were also prepared. Using RNA-sequencing, transcriptome comparisons were performed, which identified 966, 3,543 and 4,944 differentially-expressed genes (DEGs) in S3 vs. C3, S12 vs. C12 and S24 vs. C24, respectively. Gene Ontology analysis of the DEGs, revealed that treatment with GSPs is associated with disruption of the cell cycle (CC) in PC cells. Additionally, disruption of transcription, DNA replication and DNA repair were associated with GSP-treatment in PC cells. Network analysis demonstrated that the common DEGs involved in the $\mathrm{CC}$, transcription, DNA replication and DNA repair were integrated, and served essential roles in the control of CC progression in cancer cells. In summary, GSPs may exhibit a potential chemotherapeutic effect on PC cell proliferation.
\end{abstract}

\section{Introduction}

Pancreatic cancer (PC) is one of the most common causes of cancer-associated mortality in humans worldwide (1). At present, the therapeutic efficacy of PC treatment is far from satisfactory (2). Only $20 \%$ of patients with PC are eligible for surgical resection (3). The overall 5-year survival rate for

Correspondence to: Professor Zhanjiang Han, Department of Food Science, College of Life Sciences, Tarim University, 705 Hongqiao South Road, Alar, Xinjiang 843300, P.R. China

E-mail: hanzhanjiang@163.com

Key words: grape seed proanthocyanidins, pancreatic cancer, RNA sequencing, cell cycle, transcriptome, cell proliferation patients with PC is $<5 \%$ (4). To improve the cure rate for PC, it is necessary to develop improved therapeutic strategies that target the molecules associated with pancreatic tumor growth.

Chemotherapy is an effective strategy for controlling various cancer types $(5,6)$. However, natural plant products may provide promising novel options for the development of effective chemotherapeutic strategies for various cancer types (7). Proanthocyanidins are oligomeric and polymeric end-products of the flavonoid biosynthetic pathway and are widespread in natural products, including fruits, vegetables, seeds, nuts, flowers and bark $(8,9)$. Grape seeds are a particularly rich source of proanthocyanidins (10). Previous studies demonstrated the potent free radical scavenger ability of grape seed proanthocyanidins (GSPs) and GSP extract in vitro and in vivo $(11,12)$. Additionally, GSPs possess antioxidant properties and their promising anticancer effect has attracted attention in numerous studies $(13,14)$.

GSPs can reduce the viability of human PC cells and induce $\mathrm{G} 2 / \mathrm{M}$ phase cell cycle (CC) arrest in vitro, which results in apoptosis in a dose- and time-dependent manner (15). It has been demonstrated that treatment with GSPs inhibits the migration capacity of PC cells, which is associated with a decrease in nuclear factor- $\kappa \mathrm{B}(\mathrm{NF}-\kappa \mathrm{B})$ expression and reversal of the epithelial-mesenchymal transition process (16). In addition to PC cells, GSPs have been identified to inhibit the migration and invasive potential of melanoma (17), non-small cell lung cancer (18) and head and neck squamous cell carcinoma cells in in vitro cell culture models (19).

Although numerous studies have investigated the anticancer effect of proanthocyanidins (20), to the best of our knowledge, the global gene expression in response to treatment and the pathway network regulated by proanthocyanidins in cancer cells have not been thoroughly investigated. Next-generation sequencing has revealed a substantial amount of information regarding gene expression at the transcriptome level and the underlying molecular events in response to drug exposure or radiation (21). The present study used RNA-sequencing (RNA-seq) to identify the genes associated with the effects of treatment with GSPs in PC cells. Numerous differentially-expressed genes (DEGs) were revealed at different treatment exposure times and bioinformatic analysis 
identified that a number of DEGs were associated with the CC, regulation of transcription, DNA replication and DNA repair, which may explain the cytotoxicity of GSPs in PC cells.

\section{Materials and methods}

Cell culture. The human PC cell line PANC-1 was obtained from Procell (http://www.procell.com.cn; Wuhan, China) and cultured in monolayers in Dulbecco's modified Eagle's medium (Invitrogen; Thermo Fisher Scientific, Inc., Waltham, MA, USA) supplemented with 10\% fetal bovine serum (Hyclone; GE Healthcare Life Sciences, Logan, UT, USA) in a humidified incubator at $37^{\circ} \mathrm{C}$ and a $5 \% \mathrm{CO}_{2}$ atmosphere. The GSP extract, obtained from JF-NATURAL (Tianjin, China; cat. no. J011003), contained monomeric (9.5\%), dimeric (12.8\%), trimeric $(76.7 \%)$ and oligomeric (1\%) procyanidins. The $100 \mu \mathrm{g}$ GSPs extract was dissolved in $100 \mu \mathrm{l}$ dimethylsulfoxide (DMSO; Sigma-Aldrich; Merck KGaA, Darmstadt, Germany) for 10 min at room temperature prior to addition to the cell culture media. The maximum concentration of DMSO in the media did not exceed $0.1 \%$. PANC-1 cells were treated with $20 \mu \mathrm{g} / \mathrm{ml} \mathrm{GSP}$ for 3,12 and $24 \mathrm{~h}$ at $37^{\circ} \mathrm{C}$. Additionally, cells were treated with DMSO for 3,12 and $24 \mathrm{~h}$ at $37^{\circ} \mathrm{C}$ served as controls.

Cell viability assay. GSP-treated PANC-1 cells were plated in 96-well cell culture plates at $5 \times 10^{3}$ cells/well and incubated for $24 \mathrm{~h}$ at $37^{\circ} \mathrm{C}$. Subsequently, $50 \mu 1 \mathrm{MTT}$ solution $(5 \mathrm{mg} / \mathrm{ml}$; Sigma-Aldrich; Merck KGaA) was added to each well and the cells were incubated for a further $4 \mathrm{~h}$ at $37^{\circ} \mathrm{C}$. Following $3 \mathrm{~min}$ centrifugation at $5,500 \mathrm{xg}$ at $4{ }^{\circ} \mathrm{C}$, the supernatant was removed from each well. The colored formazan crystals produced by MTT in each well were dissolved in $150 \mu 1 \mathrm{DMSO}$ and the optical density values were measured at $490 \mathrm{~nm}$.

Flow cytometry. GSP-induced apoptosis in PC cells was determined by flow cytometry using an Annexin V-fluorescein isothiocyanate (FITC) Apoptosis detection kit (BD Biosciences, Franklin Lakes, NJ, USA). Following treatment with GSPs for $48 \mathrm{~h}$ at $37^{\circ} \mathrm{C}$, cells $\left(2 \times 10^{5}\right)$ were harvested, washed twice with PBS and incubated with Annexin V-FITC and propidium iodide for $10 \mathrm{~min}$ in the dark at room temperature. The stained cells were then detected and analyzed by a MoFLO XDP flow cytometer (Beckman Coulter, Inc., Brea, CA, USA) and the Cell Quest 3.3 software (BD Biosciences).

RNA extraction and sequencing. Total RNA was extracted from the GSP- or DMSO-treated PANC-1 cells using TRIzol ${ }^{\circledR}$ reagent (Invitrogen; Thermo Fisher Scientific, Inc.). The RNA was further purified by two $15 \mathrm{~min}$ phenol-chloroform (1:1) (Beijing Solarbio Science \& Technology Co., Ltd., Beijing, China) at $4^{\circ} \mathrm{C}$ treatments and then treated with RQ1DNase (Promega Corporation, Madison, WI, USA) for $30 \mathrm{~min}$ at $37^{\circ} \mathrm{C}$ to remove DNA. The quality and quantity of the purified RNA were monitored by measuring the absorbance at 260 and $280 \mathrm{~nm}$, and the A260:A280 ratio using a Smartspec Plus Spectrophotometer (Bio-Rad Laboratories, Inc., Hercules, CA, USA). The integrity of RNA was verified by $1.5 \%$ agarose gel electrophoresis.

For each sample, $10 \mu \mathrm{g}$ total RNA was used for RNA-seq library preparation. Polyadenylated mRNAs were purified and concentrated with oligo(dT)-conjugated magnetic beads
(Invitrogen; Thermo Fisher Scientific, Inc.), according to the manufacturer's protocol prior to directional RNA-seq library preparation. The libraries were prepared using the purified mRNAs by the TruSeq Stranded Total RNA LT Sample Prep Kit (Illumina, Inc., San Diego, CA, USA), according to the the manufacturer's protocol. The Nextseq 500 system (Illumina, Inc., San Diego, CA, USA) was used to collect data from 151-base pair pair-end sequencing from ABlife Inc. (https:// www.ablife.cc; Wuhan, China).

Bioinformatic analysis. Using TopHat 2.0 software (22), reads were successfully mapped against the current human reference genome (GRCH38). Reads per kilobase per million mapped reads (RPKM) was used to calculate the expression level of genes. To measure the RPKM value and screen out the DEGs, the edgeR 3.22 software package of Bioconductor software (23) was used to specifically analyze the differential expression of genes using RNA-seq data. The genes in every sample with RPKM values $<0.1$ were removed prior to analysis. A fold change $(\mathrm{FC}) \geq 2$ or $\leq 0.5$ and $\mathrm{P} \leq 0.01$ indicated a statistically significant DEG.

To predict the gene function and calculate the functional category distribution frequency, Gene Ontology (GO) and Kyoto Encyclopedia of Genes and Genomes (KEGG) analysis was employed using the DAVID bioinformatics database (https://david.ncifcrf.gov) (24). Networks were constructed by calculating the Pearson's correlation coefficient (PCC) for the expression levels of the DEGs. Cytoscape 3.5.1 software was used to display the co-expression network (25).

Validation of DEGs by reverse transcription-quantitative polymerase chain reaction ( $R T-q P C R)$. To validate the RNA-seq data, RT-qPCR was performed for selected DEGs and normalization was achieved with the human reference gene GAPDH. The primers used are presented in Table I. The same RNA samples for RNA-seq were used for RT-qPCR, and RNA extraction was performed in accordance with the aforementioned protocol and materials. In each pooled sample, $1 \mu \mathrm{g}$ total RNA was reverse transcribed using a PrimeScript ${ }^{\mathrm{TM}}$ RT Reagent kit (Takara Bio, Inc., Otsu, Japan), according to the manufacturer's protocol. qPCR was performed with a S1000 Thermal Cycler (Bio-Rad Laboratories, Inc.) and Bestar SYBR ${ }^{\circledR}$ Green RT-PCR Master mix (DBI Bioscience, Shanghai, China), according to the manufacturer's protocols. The following thermocycling conditions were used: $95^{\circ} \mathrm{C}$ for $10 \mathrm{~min}$, followed by 38 cycles of $95^{\circ} \mathrm{C}$ for $15 \mathrm{sec}$ and $60^{\circ} \mathrm{C}$ for $1 \mathrm{~min}$. PCR amplifications were performed in triplicate for each sample, and the results were quantified by $2^{-\Delta \Delta C q}$ method (26).

Statistical analysis. The data were analyzed using Microsoft Excel 2007 software (https://www.microsoft.com; Microsoft Corporation, Redmond, WA, USA). All data are presented as the mean \pm standard deviation. For comparisons between two groups, statistically significant differences between means were identified by paired Student's t-test. For multiple comparisons, the significance was determined by simple one-way analysis of variance followed by a Tukey's post-hoc test. $\mathrm{P}<0.05$ was considered to indicate a statistically significant difference.

Online data deposition. The datasets (GSE85610) generated in the present study are available from the National Center for 
Table I. Genes and primers used for reverse transcription-polymerase chain reaction.

\begin{tabular}{lll} 
Gene & \multicolumn{1}{c}{ Forward primer (5'-3') } & Reverse primer $\left(5^{\prime}-3^{\prime}\right)$ \\
\hline ATR & ACAGTTGGTGACAGTGCTT & CCTTGAGTTTCAGTTGTTGAGA \\
RAD50 & ACATCAGAAGTTGGAAGAGAAC & AGCATCCCGAAATTGTGGTT \\
NBN & TCTATCTGAGAATGAGCCTGTG & GTGTCCTTGAATAACTGTTCCA \\
BLM & CAAGAGATGTGACTGACGATGT & ATCTTCCAGAAGGACCTACATG \\
CDK1 & CCGCAACAGGGAAGAACAG & AAGCCAAGATAAGCAACTCCTT \\
MDM2 & AACGCCACAAATCTGATAGT & GATTCACTGCTACTGCTTCTT \\
HIF1A & CGTTCCTTCGATCAGTTGTCA & GTAGTGGTGGCATTAGCAGTAG \\
E2F3 & GTTCTTTGTTGTGGCTCTTGTT & TCCTTCATATCCCTCCTTCTCA \\
GAPDH & GGTCGGAGTCAACGGATTTG & GGAAGATGGTGATGGGATTTC
\end{tabular}

ATR, ataxia telangiectasia and Rad3-related; RAD50, RAD50 double strand break repair protein; NBN, nibrin; BLM, bloom syndrome RecQ like helicase; CDK1, cyclin dependent kinase 1; MDM2, mouse double minute 2 homolog; HIF1A, hypoxia inducible factor $1 \alpha$ subunit; E2F3, E2F transcription factor 3.

A

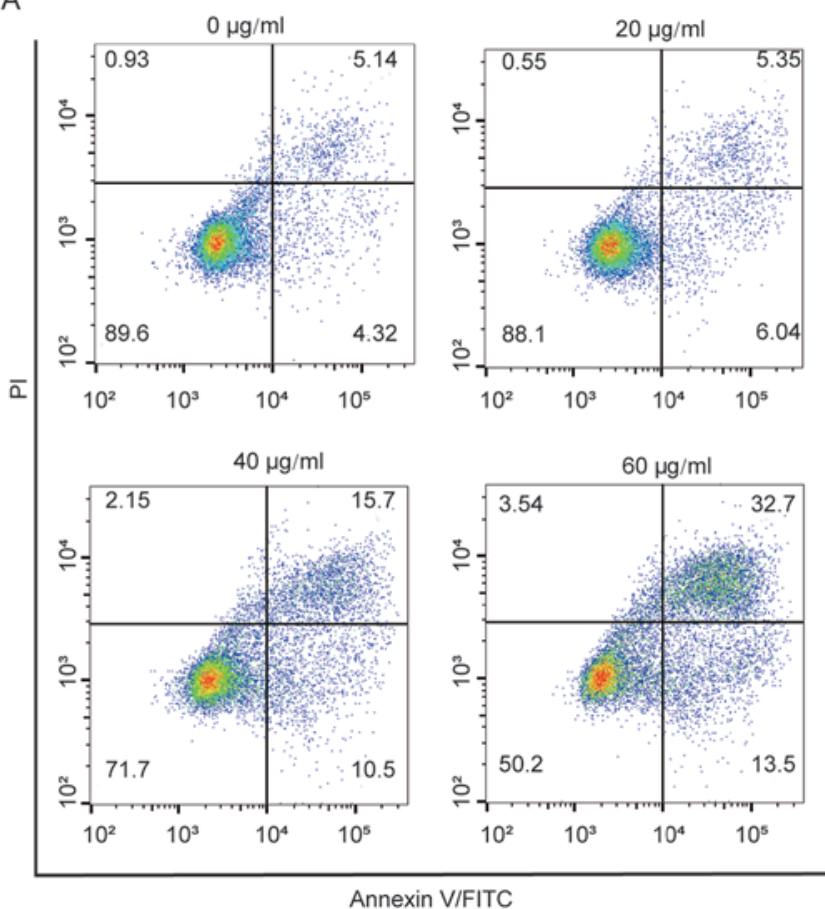

B
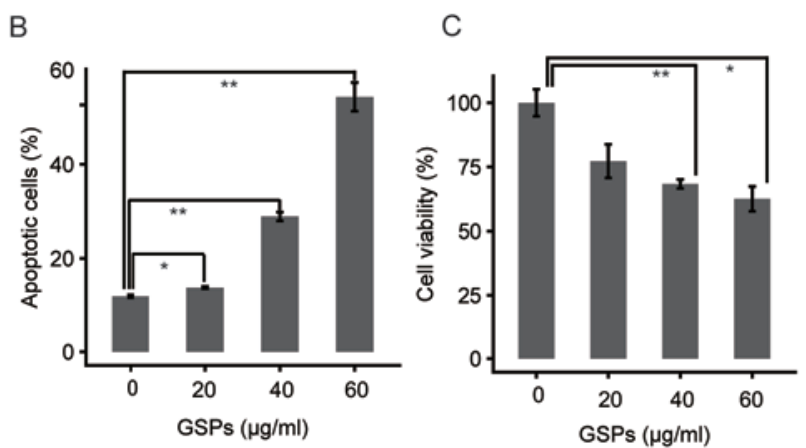

D

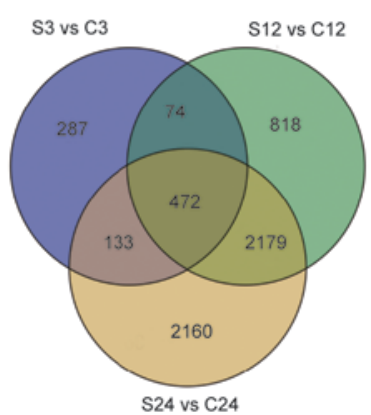

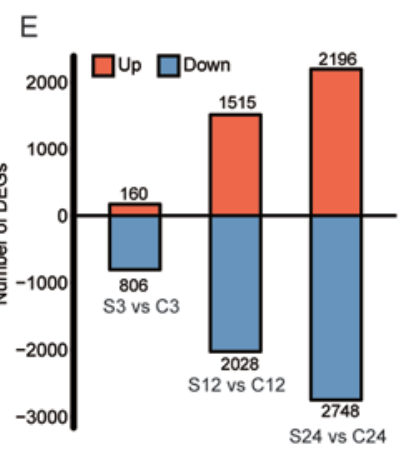

Figure 1. Determination of DEGs in PC cells following treatment with GSPs. (A) Treatment with GSPs induces apoptosis of PANC-1 cells in a dose-dependent manner. Cells were treated with various concentrations of GSPs and harvested at $12 \mathrm{~h}$ post-treatment for assessment of cellular apoptosis using Annexin V-fluorescein isothiocyanate staining coupled with flow cytometry. The upper left, upper right and lower right quadrants represent necrotic, late apoptotic and early apoptotic cells , respectively; while lower left quadrant indicates normal cells. (B) Total percentage of apoptotic PC cells in each treatment group are summarized with data presented as the mean \pm SD of two independent experiments. (C) Treatment with GSPs reduces the viability of PC cells. The viability of PC cells was determined with an MTT assay. The data are reported as the percentage relative to the percentage of control cells and presented as the mean \pm SD of three replicates. (D) Venn diagrams of the DEGs identified in the S3 vs. C3, S12 vs. C12 and S24 vs. C24 comparisons. (E) The number of upregulated and downregulated DEGs in the three comparisons. Data are presented as the mean \pm SD. Statistical analysis was performed using one-way analysis of variance followed by Tukey's post hoc test. "P<0.05 and ${ }^{* *} \mathrm{P}<0.01$. DEG, differentially expressed gene; PC, pancreatic cancer; GSP, grape seed proanthocyanidin; SD, standard deviation; PI, propidium iodide; PE, phycoerythrin.

Biotechnology Information Gene Expression Omnibus database (http://www.ncbi.nlm.nih.gov/geo/).

\section{Results}

GSPs inhibited the viability of PC cells in vitro. Using flow cytometry, the present study identified that treatment with 0,20 ,
40 or $60 \mu \mathrm{g} / \mathrm{ml}$ GSP significantly induced apoptosis of the PC cell line PANC-1 in a dose-dependent manner (Fig. 1A and B). Additionally, the effect of treatment with GSPs on the viability of PC cells was determined with an MTT assay. A significant dose-dependent decrease in the viability of PC cells was revealed $24 \mathrm{~h}$ after treatment (Fig. 1C) Furthermore, it was identified that treatment with GSPs was associated with 
Table II. Reads obtained from sequencing.

\begin{tabular}{lcccc}
\hline Sample & Raw reads & Clean reads & Total mapped reads & Unique mapped reads \\
\hline C3-A & $52,943,434$ & $44,154,519$ & $39,447,474$ & $36,610,491$ \\
S3-A & $51,800,450$ & $40,793,788$ & $35,894,276$ & $33,010,744$ \\
C12-A & $47,942,930$ & $36,813,374$ & $31,477,445$ & $28,953,208$ \\
S12-A & $51,594,804$ & $4,812,991$ & $35,626,805$ & $32,558,389$ \\
C24-A & $52,301,536$ & $41,559,257$ & $36,042,733$ & $33,192,336$ \\
S24-A & 68130,442 & $48,397,199$ & $40,704,353$ & $36,919,068$ \\
C3-B & $55,301,368$ & $45,043,351$ & $3,981,052$ & $36,977,763$ \\
S3-B & $59,484,624$ & $46,200,745$ & $40,600,169$ & $37,426,268$ \\
C12-B & $54,587,246$ & $42,500,995$ & $36,816,554$ & $34,184,032$ \\
S12-B & $47,518,592$ & $37,483,400$ & $32,481,709$ & $29,620,467$ \\
C24-B & $51,110,770$ & $40,435,967$ & $35,338,949$ & $32,542,109$ \\
S24-B & $62,127,244$ & $4,6691,863$ & $40,212,872$ & $36,466,759$ \\
\hline
\end{tabular}

necrosis (upper left quadrants) only in a small number of PC cells in a dose-dependent manner (Fig. 1A).

Analysis of RNA-seq data and determination of DEGs. To investigate how GSPs regulate gene expression and avoid high levels of necrotic cells, PANC-1 cells were treated with $20 \mu \mathrm{g} / \mathrm{ml} \mathrm{GSP}$ for 3, 12 and $24 \mathrm{~h}$, and the RNA samples termed S3, S12 and S24 were prepared, respectively. Cells treated with DMSO for 3, 12 and $24 \mathrm{~h}$ served as controls and the control RNA samples termed C3, C12 and C24 were prepared. Two biological replicates were generated at each time point, therefore, a total of 12 cDNA libraries (S3-A, S3-B, C3-A, C3-B, S12-A, S12-B, C12-A, C12-B, S24-A, S24-B, C24-A and C24-B) were constructed for RNA-seq.

Using Illumina NextSeq 500, >0.65 billion pair-end reads were generated; corresponding to $\sim 54.6$ million sequence reads per sample (Table II). Using TopHat 2.0 software (22), $68.4 \%$ of all reads were successfully mapped against the current human reference genome (GRCH38).

Using edgeR 22.0 software (23), a total of 966, 3,543 and 4,944 statistically significant DEGs were identified in S3 vs. C3, S12 vs. S12 and S24 vs. C24, respectively (Fig. 1D and E), which indicates marked changes to the transcriptome in PANC-1 cells following treatment with GSPs. Additionally, 456 common DEGs were identified between all three comparisons.

Treatment with GSPs disrupts the CC of PC cells. To identify the primary functions in which the DEGs are involved, GO enrichment analysis was performed (Fig. 2). A total of 24, 52 and $64 \mathrm{GO}$ terms were identified in S3 vs. C3, S12 vs. $\mathrm{C} 12$ and $\mathrm{S} 24$ vs. C24, respectively ( $\mathrm{P}<0.05$; data not shown).

For all three comparisons, numerous DEGs were enriched in the following terms: 'Mitotic cell cycle' (GO:0000278), 'G2/M transition of mitotic cell cycle' (GO:0000086), 'mitosis' (GO:0007067) and 'cell cycle' (GO:0007049) (Fig. 2B, D and F). This indicates that treatment with GSPs exhibits an effect on the CC of PANC-1 cells. To determine how GSPs affect the CC of PANC-1 cells, the expression levels of DEGs enriched in the aforementioned GO terms were analyzed by RNA-seq. This demonstrated that the expression levels of a number of DEGs associated with the $\mathrm{CC}$, including cyclin dependent kinase 1 (CDK1) and mouse double minute 2 homolog (MDM2), decreased in GSP-treated cells, compared with the control cells (Fig. 3). CDK1 serves a key role in the eukaryotic $\mathrm{CC}$ by regulating the centrosome cycle and the onset of mitosis, and modulating the G2-M transition, G1 progression and G1-S transition via an association with multiple interphase cyclins, including CDC7, CDC20 and CDC25A (27). MDM2 can inhibit CC arrest and apoptosis by binding to transcriptional activation domain of TP53 (28).

The results of RT-qPCR were consistent with the results of RNA-seq (Fig. 3B). PCC values were $>0.9$, which indicates that treatment with GSPs may disrupt the CC of PANC-1 cells. Additionally, certain genes associated with the $\mathrm{CC}$ were identified to be upregulated in cells treated with GSPs, compared with control cells. For example, the mitogen-activated protein kinase 3 (MAPK3) gene was revealed as an upregulated DEG in S12 vs. C12 and S24 vs. C24 (data not shown). MAPK3 serves a role in a signaling cascade that mediates various cellular processes, including proliferation, differentiation and CC progression, in response to a variety of extracellular signals (29).

Treatment with GSPs disrupts the transcription of PC cells. There are intricate connections between $C C$ regulation and transcriptional control, and progression of the CC is partially controlled at the transcriptional level in eukaryotes (30). Therefore, disruption of the transcription machinery may induce disruption of the $\mathrm{CC}$.

$\mathrm{GO}$ analysis revealed that the DEGs identified in $\mathrm{S} 3$ vs. C3, S12 vs. $\mathrm{C} 12$ and S24 vs. C24 were enriched in the 'regulation of transcription, DNA-dependent' (GO:0006355) and 'transcription, DNA-dependent' (GO:0006351) terms (Fig. 2B, D and F). This indicates that normal regulation of transcription may be disrupted in PANC-1 cells following treatment with GSPs. Furthermore, the expression levels of multiple DEGs associated with transcriptional regulation, including E2F transcription factor 3 (E2F3) and hypoxia inducible factor $1 \alpha$ subunit (HIF1A), decreased in GSP-treated cells, compared with control cells (Fig. 3). E2F3 is a transcription factor that recognizes a specific sequence motif 
A

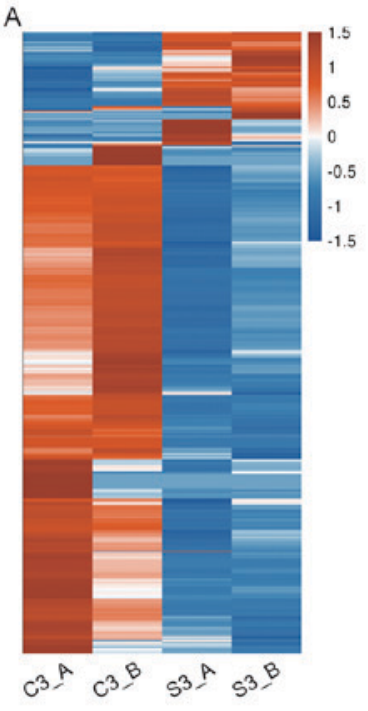

C

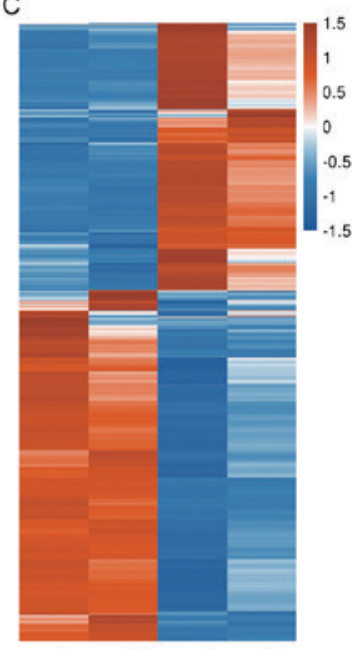

$$
0^{12}{ }^{P} C^{12^{B}} 5^{12^{P}} 5^{12^{B}}
$$

E

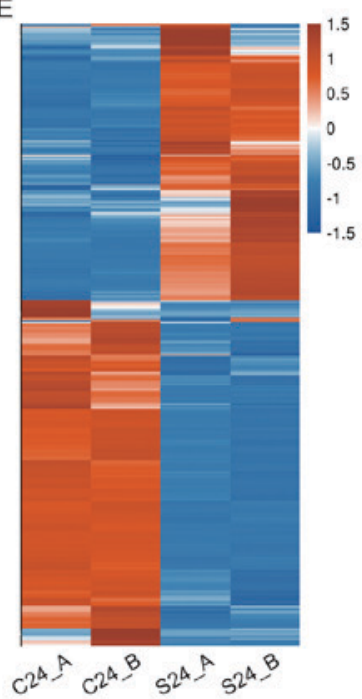

B

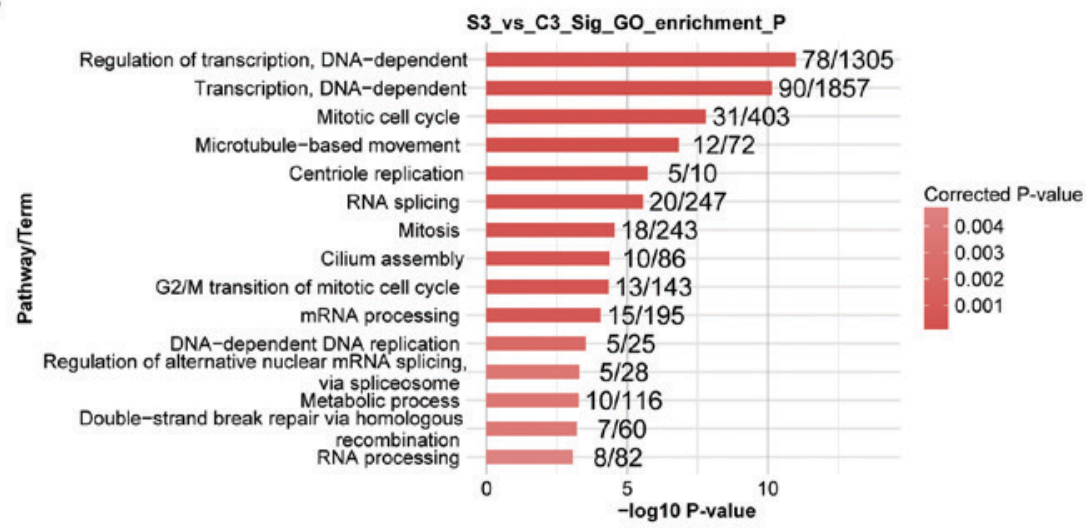

D

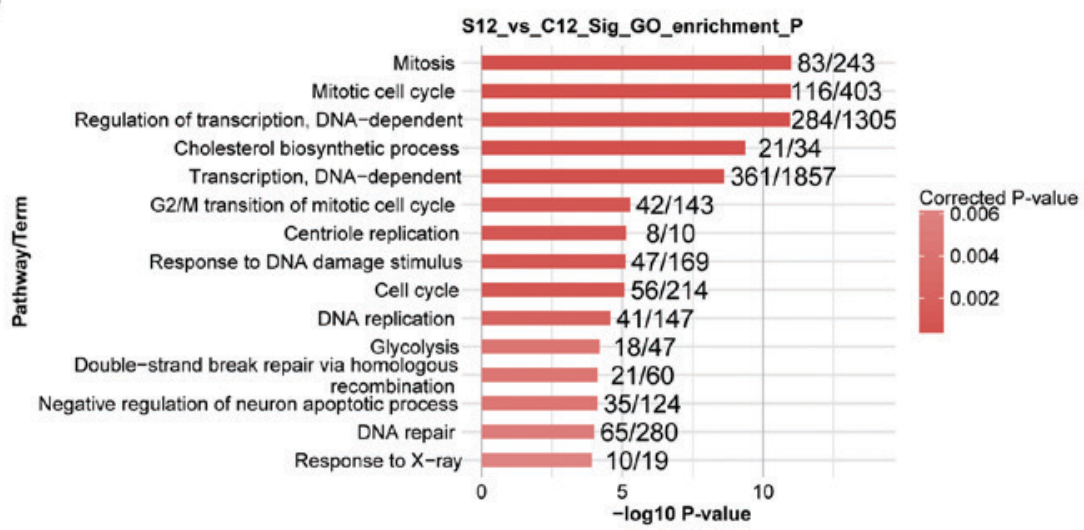

F

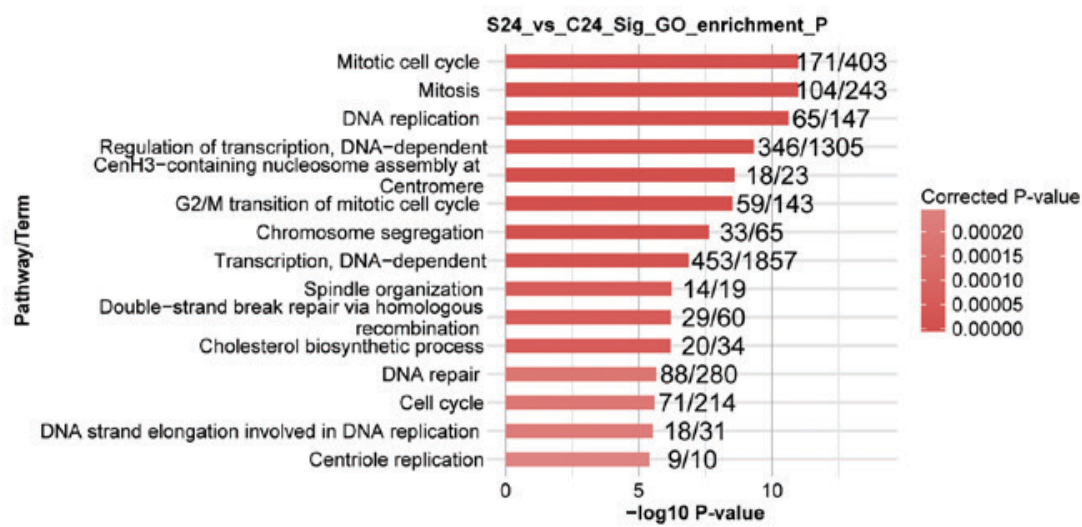

Figure 2. GO analysis of DEGs from S3 vs. C3, S12 vs. C12 and S24 vs. C24. (A) Heat maps and (B) GO analysis of DEGs from S3 vs. C3. (C) Heat maps and (D) GO analysis of DEGs from S12 vs. C12. (E) Heat maps and (F) GO analysis of DEGs from S24 vs. C24. The heat maps were generated by hierarchical analysis of genes and samples, and red and blue represented upregulated and downregulated DEGs, respectively. The top 15 terms are presented. GO, Gene Ontology; DEG, differentially expressed gene.

in DNA and interacts directly with the retinoblastoma protein to modulate the expression of genes involved in the CC (31).
HIF1A functions as a master transcriptional regulator of the adaptive response to hypoxia, which serves as a stimulus for 

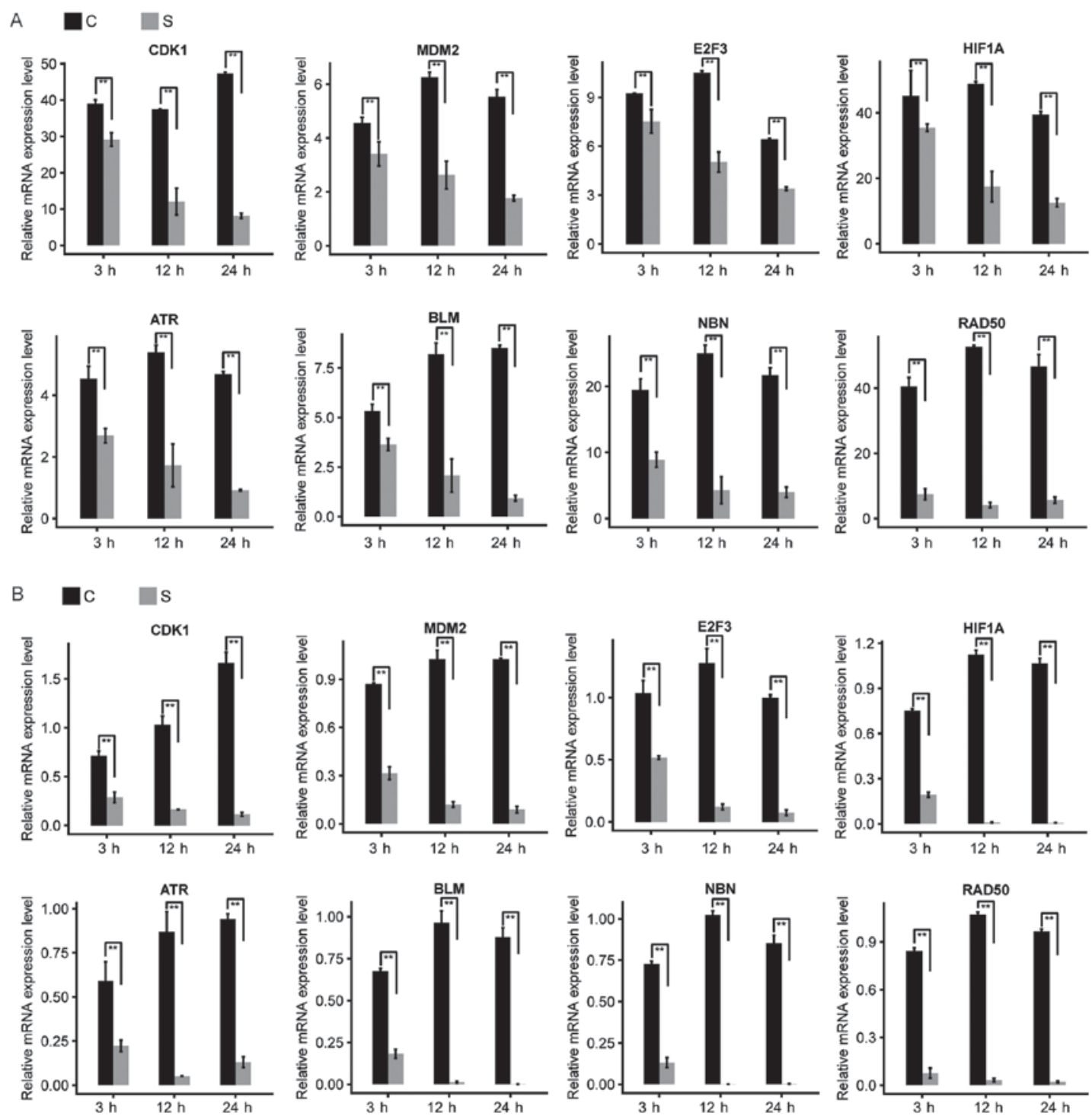

Figure 3. RT-qPCR validation of certain DEGs identified by RNA-sequencing in GSPs-treatment (S) and control (C) samples. (A) mRNA expression levels of certain DEGs were determined by high-throughput sequencing. Reads per kilobase per million mapped reads was used to calculate the expression levels of genes. (B) The mRNA expression levels of the same DEGs were validated by RT-qPCR and normalized against GAPDH expression level. Statistical analysis was performed by Student's t-test and data are presented as the mean \pm standard deviation and experiment was performed in triplicate. ${ }^{* *} \mathrm{P}<0.01$. RT-qPCR, reverse transcription-quantitative polymerase chain reaction; DEG, differentially expressed gene; CDK1, cyclin dependent kinase 1; MDM2, mouse double minute 2 homolog; E2F3, E2F transcription factor 3; HIF1A, hypoxia inducible factor $1 \alpha$ subunit; BLM, bloom syndrome RecQ like helicase; NBN, nibrin; RAD50, RAD50 double strand break repair protein.

CC arrest (32). A number of genes associated with regulation of transcription were also identified to be upregulated in GSP-treated cells, compared with control cells. For example, JunB proto-oncogene, a family member of the activator protein 1 transcription factors, was identified as an upregulated DEG in S3 vs. C3, S12 vs. C12 and S24 vs. C24 (data not shown). These observations indicate that the transcriptional machinery in PANC-1 cells may be disrupted following treatment with GSPs, which may result in disruption of the CC.

Treatment with GSPs disrupts DNA replication and repair. Accurate DNA replication and repair are crucial in the normal CC. The DEGs identified in S3 vs. C3, S12 vs. C12 and S24 vs. C24 were enriched in the 'DNA-dependent DNA replication' (GO: 0006261), 'DNA replication' (GO: 0006260), 'DNA repair' (GO: 0006281) and 'double-strand break repair via homologous recombination' (GO: 0000724) GO terms (Fig. 2B, D and F). The expression levels of numerous DEGs enriched in the aforementioned terms, including ataxia telangiectasia and Rad3-related (ATR), bloom syndrome RecQ like helicase (BLM), nibrin (NBN) and RAD50 double strand break repair protein (RAD50), were decreased in the PANC-1 cells treated with GSPs, compared with the control cells (Fig. 3). ATR serves a critical role in the DNA damage response (33). NBN serves a role in the control of the intra-S-phase checkpoint (34). Additionally, evidence indicates that $\mathrm{NBN}$ is involved in the G1 and G2 checkpoints, and is involved in DNA double-strand break repair and DNA damage-induced checkpoint activation (35). BLM serves a role in DNA replication and repair (36). RAD50, in cooperation with other molecules, is important in DNA double-strand break repair, $\mathrm{CC}$ checkpoint activation, telomere maintenance 


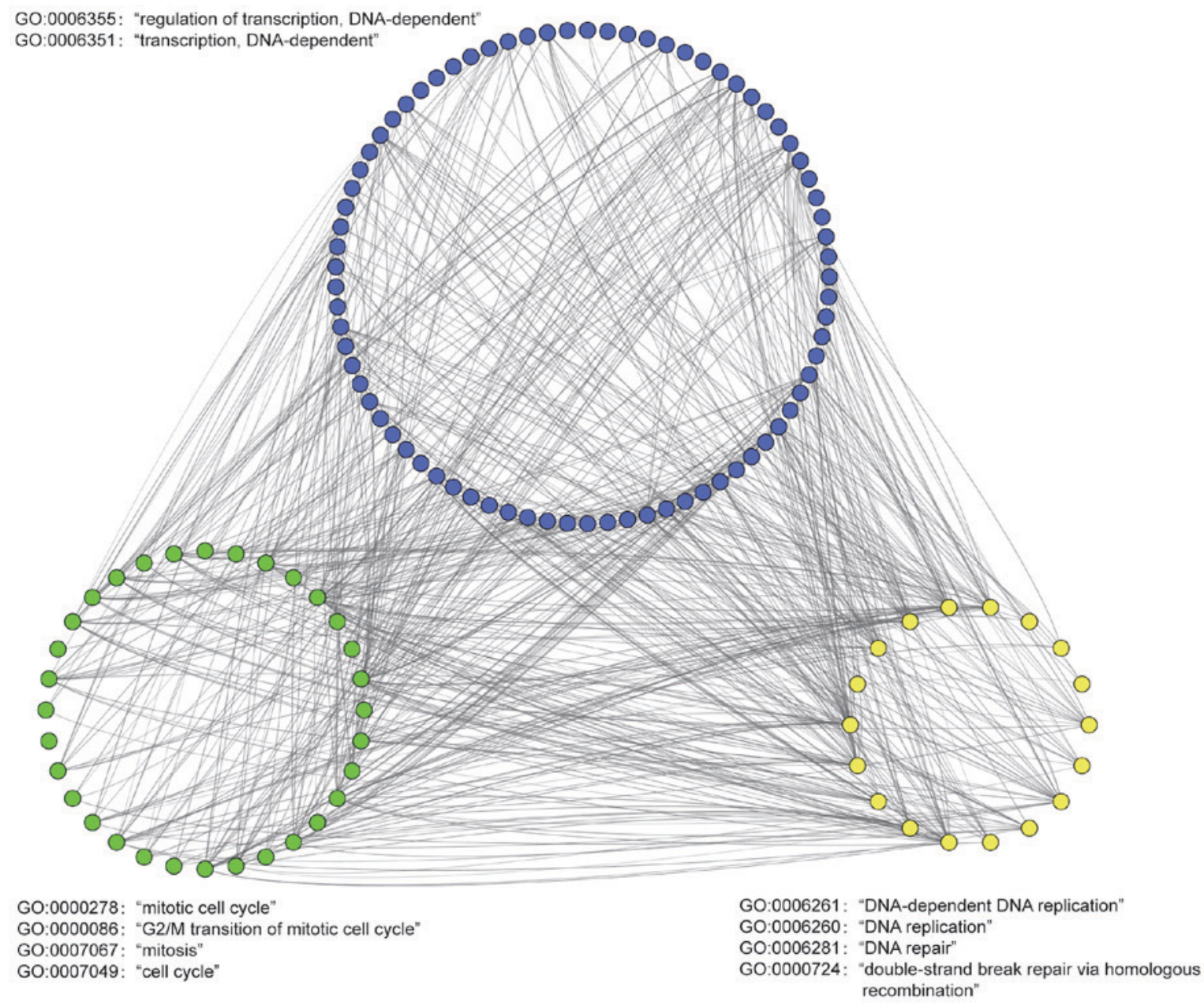

Figure 4. Co-expression analysis of DEGs associated with regulation of the cell cycle. Network analysis was performed using the Pearson correlation coefficients of the common DEGs enriched in 'mitotic cell cycle' (GO:0000278), 'G2/M transition of mitotic cell cycle' (GO:0000086), 'mitosis' (GO:0007067), 'cell cycle' (GO:0007049), 'regulation of transcription, DNA-dependent' (GO:0006355), 'transcription, DNA-dependent' (GO:0006351), 'DNA-dependent DNA replication' (GO:0006261), 'DNA replication' (GO:0006260), 'DNA repair' (GO:0006281) and 'double-strand break repair via homologous recombination' (GO:0000724). The nodes represent DEGs and the lines signify significant correlations. $\mathrm{PCC} \geq 0.95$ and $\mathrm{P}<0.01$ indicated a statistically significant correlation. DEG, differentially expressed gene.

and meiotic recombination (37). A number of genes associated with DNA replication and repair were also identified to be upregulated in GSP-treated cells, compared with control cells. For example, the ubiquitin $\mathrm{C}$ gene, which serves an important role in DNA repair (38), was revealed to be an upregulated DEG in S12 vs. C12 and S24 vs. C24 (data not shown). These observations indicate that the regulation of DNA replication and repair may be disrupted in PANC-1 cells following treatment with GSPs, which may result in disruption of the CC.

Network analysis of DEGs reveals that treatment with GSPs induces apoptosis. The aforementioned results revealed that disruption of transcription and DNA replication may affect the CC of PC cells, and induce apoptosis of cancer cells. To determine the co-expression associations of the DEGs, a network was constructed using the PCCs of the common DEGs ( $\mathrm{PPC} \geq 0.95 ; \mathrm{P}<0.01$ ), which were enriched in the 'mitotic cell cycle' (GO:0000278), 'G2/M transition of mitotic cell cycle' (GO:0000086), 'mitosis' (GO:0007067), 'cell cycle' (GO:0007049), 'regulation of transcription, DNA-dependent' (GO:0006355), 'transcription, DNA-dependent' (GO:0006351), 'DNA-dependent DNA replication' (GO:0006261), 'DNA replication' (GO:0006260), 'DNA repair' (GO:0006281) and 'double-strand break repair via homologous recombination' (GO:0000724) pathways (Fig. 4). The co-expression associations between these DEGs indicated that disorders of transcription, DNA replication, DNA repair and CC worked together to induce apoptosis, resulting in the anticancer effects of GSPs.

\section{Discussion}

GSPs are biologically active components of grape seeds and have been demonstrated to possess a wide array of pharmacological and biochemical properties (39). Previous studies revealed that GSPs exhibit inhibitory effects on various cancer types, including PC $(15,16,40)$, and certain molecular mechanisms of the anticancer effects have been identified (41). It has been revealed that treatment with GSPs induces apoptosis mediated by the mitochondrial-pathway in human colorectal carcinoma cells (42). It has also been demonstrated that GSPs can inhibit the proliferation, migration and invasion of tongue squamous cell carcinoma cells by suppressing the protein kinase $\mathrm{B} / \mathrm{NF}-\mathrm{\kappa B}$ signaling pathway (43). 
In the present study, RNA-seq analysis indicated that treatment with GSPs did not affect the mitochondrial-pathway and the protein kinase $\mathrm{B} / \mathrm{NF}-\kappa \mathrm{B}$ signaling pathway; however, the expression levels of numerous genes involved in the regulation of the CC were markedly affected in PC cells. This observation was consistent with previous studies that have indicated that treatment with GSPs can upregulate and downregulate gene expression in certain functional pathways, including MAPK and cytokinesis pathways (44). Furthermore, the results of functional analysis of DEGs were consistent with those of previous studies $(15,44)$ and more targeted pathways were identified, including specific pathways associated with cell metabolism. For example, 'metabolic process' (GO:0008152) was identified in S3 vs. C3, while 'glycolysis' (GO:0006096) and 'cholesterol biosynthetic process' (GO:0006695) were revealed in S12 vs. C12 and S24 vs. C24. These observations indicate that GSPs have the capacity to affect numerous aspects of PC cells and GSPs are promising bioactive molecules for blocking the proliferation of cancer cells.

In the normal $\mathrm{CC}$, the control of gene expression timing is of great importance and deregulation of the timing can be detrimental (45). Numerous factors, including transcription factors, kinases, DNA polymerase, kinesins and cyclins, require precise timing of expression for their function in $\mathrm{CC}$ regulation (46) and dysregulation of the expression of these factors may inhibit cell proliferation. For example, it has previously been identified that oligomeric proanthocyanidins target cancer stem-like cells and suppress tumor organoid formation in colorectal cancer by inhibiting the Hippo pathway through downregulation of its key transcriptional regulators, yes-associated protein (YAP) and tafazzin (TAZ) (47). In the present study, treatment with GSPs also affected the expression levels of multiple genes involved in the regulation of transcription in PC cells, indicating that the timing of normal transcription may be disrupted during the CC. However, YAP and TAZ were not revealed as DEGs in S3 vs. C3, S12 vs. C12 and S24 vs. C24, and results of KEGG analysis indicated that 'hippo signaling pathway' (ID: hsa04390) was not enriched in the three comparisons (data not shown). It has been indicated that treatment with GSPs may disturb normal transcription through different molecular mechanisms in different types of cancer cell. Therefore, in the present study, apoptosis of PC cells may be partially induced by the deregulation of transcription through novel mechanisms that had not been reported in previous studies.

It is understood that identical genetic information must be precisely transmitted from the mother cell to the daughter cells during successive cell proliferation cycles (48). If a cell enters mitosis with partially replicated chromosomes or if a cell over-replicates its chromosomal DNA, genetic alterations could occur, causing possible cell death (49). Therefore, accurate DNA replication and repair is crucial for the normal CC, and disrupting the mechanism of DNA replication and repair is a practical strategy to inhibit the proliferation of cancer cells $(50,51)$. The present study revealed that treatment with GSPs had the capacity to interfere with the expression levels of multiple genes associated with DNA replication and repair in PC cells, indicating that these mechanisms may have been disrupted. This may be the reason why treatment with GSPs induced CC arrest and apoptosis in PC cells.

\section{Acknowledgements}

The authors would like to thank Dr Shengjie Liao and Miss Hong Wu from ABLife Inc. for their scientific input and editorial assistance.

\section{Availability of data and materials}

The datasets generated and/or analyzed during the current study are available in the Gene Expression Omnibus repository (accession no. GSE85610), https://www.ncbi.nlm.nih.gov/geo/; these data will be made publicly available on August 19, 2019.

\section{Funding}

The present study was supported by the National Natural Science Foundation of China (grant nos. 31360409, 31471667 and 31660489), Program for Young and Middle-aged Leading Talents of China Xinjiang Production and Construction Corps (grant no. 2017CB009) and the Selection and Cultivation Project of 'Talents of Xinjiang Production and Construction Corps' (grant no. 00608017).

\section{Authors' contributions}

LZ, WW and ZH designed and managed the project. WW, LZ and ZH drafted the manuscript. DG, YZ, YX and MT participated in sample collection and manuscript revision. All authors read and approved the final manuscript.

\section{Ethics approval and consent to participate}

Not applicable.

\section{Patient consent for publication}

Not applicable.

\section{Competing interests}

The authors declare that they have no competing interests.

\section{References}

1. Kamisawa T, Wood LD, Itoi T and Takaori K: Pancreatic cancer. Lancet 388: 73-85, 2016.

2. Huang X, Zhi X, Gao Y, Ta N, Jiang $H$ and Zheng J: LncRNAs in pancreatic cancer. Oncotarget 7: 57379-57390, 2016.

3. Yeo TP, Hruban RH, Leach SD, Wilentz RE, Sohn TA, Kern SE, Iacobuzio-Donahue CA, Maitra A, Goggins M, Canto MI, et al: Pancreatic cancer. Curr Probl Cancer 26: 176-275, 2002.

4. Li D, Xie K, Wolff R and Abbruzzese JL: Pancreatic cancer. Lancet 363: 1049-1057, 2004.

5. Rajkumar SV and Moreau P: Decade in review-haematological cancer: Advances in biology and therapy. Nat Rev Clin Oncol 11: 628-630, 2014.

6. Gisselbrecht $\mathrm{C}$ and Sibon D: New perspectives in the therapeutic approach of peripheral T-cell lymphoma. Curr Opin Oncol 30: 285-291, 2018.

7. Lee Y: Cancer chemopreventive potential of procyanidin. Toxicol Res 33: 273-282, 2017.

8. Bagchi D, Bagchi M, Stohs S, Ray SD, Sen CK and Preuss HG: Cellular protection with proanthocyanidins derived from grape seeds. Ann N Y Acad Sci 957: 260-270, 2002. 
9. Dixon RA, Xie DY and Sharma SB: Proanthocyanidins-a final frontier in flavonoid research? New Phytol 165: 9-28, 2005.

10. Katiyar SK: Grape seed proanthocyanidines and skin cancer prevention: Inhibition of oxidative stress and protection of immune system. Mol Nutr Food Res 52 (Suppl 1): S71-S76, 2008.

11. Bagchi D, Bagchi M, Stohs SJ, Das DK, Ray SD, Kuszynski CA, Joshi SS and Pruess HG: Free radicals and grape seed proanthocyanidin extract: Importance in human health and disease prevention. Toxicology 148: 187-197, 2000.

12. Du Y and Lou H: Catechin and proanthocyanidin B4 from grape seeds prevent doxorubicin-induced toxicity in cardiomyocytes. Eur J Pharmacol 591: 96-101, 2008

13. Rossi M, Negri E, Parpinel M, Lagiou P, Bosetti C, Talamini R, Montella M, Giacosa A, Franceschi S and La Vecchia C: Proanthocyanidins and the risk of colorectal cancer in Italy. Cancer Causes Control 21: 243-250, 2010.

14. Singh T, Sharma SD and Katiyar SK: Grape proanthocyanidins induce apoptosis by loss of mitochondrial membrane potential of human non-small cell lung cancer cells in vitro and in vivo. PLoS One 6: e27444, 2011.

15. Prasad R, Vaid M and Katiyar SK: Grape proanthocyanidin inhibit pancreatic cancer cell growth in vitro and in vivo through induction of apoptosis and by targeting the PI3K/Akt pathway. PLoS One 7: e43064, 2012.

16. Prasad R and Katiyar SK: Grape seed proanthocyanidins inhibit migration potential of pancreatic cancer cells by promoting mesenchymal-to-epithelial transition and targeting NF- $\kappa \mathrm{B}$ Cancer Lett 334: 118-126, 2013.

17. Vaid M, Singh T and Katiyar SK: Grape seed proanthocyanidins inhibit melanoma cell invasiveness by reduction of PGE2 synthesis and reversal of epithelial-to-mesenchymal transition. PLoS One 6: e21539, 2011.

18. Punathil $\mathrm{T}$ and Katiyar SK: Inhibition of non-small cell lung cancer cell migration by grape seed proanthocyanidins is mediated through the inhibition of nitric oxide, guanylate cyclase, and ERK1/2. Mol Carcinog 48: 232-242, 2009.

19. Sun Q, Prasad R, Rosenthal E and Katiyar SK: Grape seed proanthocyanidins inhibit the invasive potential of head and neck cutaneous squamous cell carcinoma cells by targeting EGFR expression and epithelial-to-mesenchymal transition. BMC Complement Altern Med 11: 134, 2011.

20. Smeriglio A, Barreca D, Bellocco E and Trombetta D Proanthocyanidins and hydrolysable tannins: Occurrence, dietary intake and pharmacological effects. Br J Pharmacol 174: 1244-1262, 2017.

21. Regier N, Beauvais-Flück R, Slaveykova VI and Cosio C: Elodea nuttallii exposure to mercury exposure under enhanced ultraviolet radiation: Effects on bioaccumulation, transcriptome, pigment content and oxidative stress. Aquat Toxicol 180: 218-226, 2016.

22. Trapnell C, Pachter L and Salzberg SL: TopHat: Discovering splice junctions with RNA-Seq. Bioinformatics 25: 1105-1111, 2009.

23. Robinson MD, McCarthy DJ and Smyth GK: edgeR: A Bioconductor package for differential expression analysis of digital gene expression data. Bioinformatics 26: 139-140, 2010.

24. Huang da W, Sherman BT and Lempicki RA: Systematic and integrative analysis of large gene lists using DAVID bioinformatics resources. Nat Protoc 4: 44-57, 2009.

25. Shannon P, Markiel A, Ozier O, Baliga NS, Wang JT, Ramage D, Amin N, Schwikowski B and Ideker T: Cytoscape: A software environment for integrated models of biomolecular interaction networks. Genome Res 13: 2498-2504, 2003.

26. Livak KJ and Schmittgen TD: Analysis of relative gene expression data using real-time quantitative PCR and the 2(-Delta Delta C(T)) method. Methods 25: 402-408, 2001.

27. Wang P, Larouche M, Normandin K, Kachaner D, Mehsen H, Emery G and Archambault V: Spatial regulation of greatwall by Cdk1 and PP2A-Tws in the cell cycle. Cell Cycle 15: 528-539, 2016.

28. Mendoza M, Mandani G and Momand J: The MDM2 gene family. Biomol Concepts 5: 9-19, 2014.

29. Liszka L: Ductal adenocarcinoma of the pancreas usually retained SMAD4 and p53 protein status as well as expression of epithelial-to-mesenchymal transition markers and cell cycle regulators at the stage of liver metastasis. Pol J Pathol 65: 100-112, 2014.

30. Dynlacht BD: Regulation of transcription by proteins that control the cell cycle. Nature 389: 149-152, 1997.
31. Ping Z, Lim R, Bashir T, Pagano $\mathrm{M}$ and Guardavaccaro D: APC/C (Cdh1) controls the proteasome-mediated degradation of E2F3 during cell cycle exit. Cell Cycle 11: 1999-2005, 2012.

32. Hubbi ME and Semenza GL: An essential role for chaperone-mediated autophagy in cell cycle progression. Autophagy 11: 850-851, 2015.

33. Zou L: DNA replication checkpoint: New ATR activator identified. Curr Biol 27: R33-R35, 2017.

34. Pennisi R, Antoccia A, Leone S, Ascenzi P and di Masi A: Hsp90 $\alpha$ regulates ATM and NBN functions in sensing and repair of DNA double-strand breaks. FEBS J 284, 2378-2395, 2017.

35. Wei L, Nakajima S, Böhm S, Bernstein KA, Shen Z, Tsang M, Levine AS and Lan L: DNA damage during the G0/G1 phase triggers RNA-templated, Cockayne syndrome B-dependent homologous recombination. PNAS 112: E3495-3504, 2015.

36. Manthei KA and Keck JL: The BLM dissolvasome in DNA replication and repair. Cell Mol Life Sci 70: 4067-4084, 2013.

37. Lafrance-Vanasse J, Williams GJ and Tainer JA: Envisioning the dynamics and flexibility of Mre11-Rad50-Nbs1 complex to decipher its roles in DNA replication and repair. Prog Biophys Mol Biol 117: 182-193, 2015.

38. Franko J, Ashley C and Xiao W: Molecular cloning and functional characterization of two murine cDNAs which encode Ubc variants involved in DNA repair and mutagenesis. Biochim Biophys Acta 1519: 70-77, 2001.

39. Katiyar SK, Pal HC and Prasad R: Dietary proanthocyanidins prevent ultraviolet radiation-induced non-melanoma skin cancer through enhanced repair of damaged DNA-dependent activation of immune sensitivity. Semin Cancer Biol 46: 138-145, 2017.

40. Chung YC, Huang CC, Chen CH, Chiang HC, Chen KB, Chen YJ, Liu CL, Chuang LT, Liu M and Hsu CP: Grape-seed procyanidins inhibit the in vitro growth and invasion of pancreatic carcinoma cells. Pancreas 41: 447-454, 2012.

41. Katiyar SK and Athar M: Grape seeds: Ripe for cancer chemoprevention. Cancer Prev Res (Phila) 6: 617-621, 2013.

42. Zhang C, Chen W, Zhang X, Zheng Y, Yu F, Liu Y and Wang Y: Grape seed proanthocyanidins induce mitochondrial pathway-mediated apoptosis in human colorectal carcinoma cells. Oncol Lett 14: 5853-5860, 2017.

43. Yang N, Gao J, Cheng X, Hou C, Yang Y, Qiu Y, Xu M, Zhang Y and Huang S: Grape seed proanthocyanidins inhibit the proliferation, migration and invasion of tongue squamous cell carcinoma cells through suppressing the protein kinase $\mathrm{B} /$ nuclear factor- $\mathrm{K}$ signaling pathway. Int J Mol Med 40: 1881-1888, 2017.

44. Chu H, Tang Q, Huang H, Hao W and Wei X: Grape-seed proanthocyanidins inhibit the lipopolysaccharide-induced inflammatory mediator expression in RAW264.7 macrophages by suppressing MAPK and NF-kb signal pathways. Environ Toxicol Pharmacol 41: 159-166, 2016.

45. Fischer M and Müller GA: Cell cycle transcription control: DREAM/MuvB and RB-E2F complexes. Crit Rev Biochem Mol Biol 52: 638-662, 2017.

46. Sadasivam S and DeCaprio JA: The DREAM complex: Master coordinator of cell cycle-dependent gene expression. Nat Rev Cancer 13: 585-595, 2013.

47. Toden S, Ravindranathan P, Gu J, Cardenas J, Yuchang M and Goel A: Oligomeric proanthocyanidins (OPCs) target cancer stem-like cells and suppress tumor organoid formation in colorectal cancer. Sci Rep 8: 3335, 2018.

48. Vermeulen K, Van Bockstaele DR and Berneman ZN: The cell cycle: A review of regulation, deregulation and therapeutic targets in cancer. Cell Prolif 36: 131-149, 2003.

49. Nishitani $\mathrm{H}$ and Lygerou Z: Control of DNA replication licensing in a cell cycle. Genes Cells 7: 523-534, 2002.

50. Murai J: Targeting DNA repair and replication stress in the treatment of ovarian cancer. Int J Clin Oncol 22: 619-628, 2017.

51. Helleday T: Amplifying tumour-specific replication lesions by DNA repair inhibitors-a new era in targeted cancer therapy. Eur J Cancer 44: 921-927, 2008.

This work is licensed under a Creative Commons Attribution-NonCommercial-NoDerivatives 4.0 International (CC BY-NC-ND 4.0) License. 